\title{
FUNCTIONAL SEQUELAE IN THE LIMBS ASSOCIATED WITH BREAST CANCER. CAN QUALITY OF LIFE QUESTIONNAIRES REPLACE OBJECTIVE CLINICAL MEASUREMENTS?
}

Jonathas José Silva', Fabiola Cristina Brandini Silva', Almir José Sarri', Idam Olivieira-Junior¹, René Aloisio Costa Vieira²

${ }^{1}$ Hospital de Câncer de Barretos - Barretos (SP), Brazil.

${ }^{2}$ Hospital de Câncer de Muriaé - Muriaé (MG), Brazil.

Introduction: Assessing limb functionality is difficult in the mastologsit's office. The growing incidence of breast cancer and consequently of cancer treatment and patient survival justifies the search for reliable quantitative evaluation methods to diagnose and correlate treatment-related chronic functional sequelae. In addition, many clinical limitations are not associated with the absolute values of strength and mobility of the upper limb. Objectives: To verify if the EORTC-BR23, SPADI and BCTOS questionnaires are able to differentiate known groups in relation to functionality. Methods: Study approved by the Ethics Committee under number 782/2014. Observational cross-sectional, non-randomized study. 48 patients treated with conservative surgery associated with radiotherapy were selected through a non-probabilistic sample for convenience and divided into two groups: Gl composed of 24 women with a minimum of two functional deficits diagnosed by goniometry and dynamometry; and G2 - women without deficits and functional complaints. Sequelae were considered a 10-degree reduction in abduction or flexion, or a $12 \%$ difference in loss of strength in the limb homolateral to breast cancer. All patients answered the SPADI, BCTOS and EORTC-BR 23 questionnaires. They sought to assess the domains of the questionnaires that would be able to separate the groups. The Man-Whitney test was used to compare the groups. If the questionnaires were able to distinguish the groups, a potential cut-off point was identified by the ROC curve. Grant by FAPESP project n. 2014 08197-0. Results: Regarding groups G1 and G2, a difference was observed in all domains of the SPADI questionnaires [pain $(\mathrm{p}<0.001)$ and disability $(\mathrm{p}<0.001)]$ and BCTOS [(functional $(\mathrm{p}=-0.04)$, cosmesis $(\mathrm{p}=0.025)$, breast pain ( $\mathrm{p}=0.053)$ and edema $(\mathrm{p}=0.007)]$, and in EORTC-BR 23 only in the "Symptoms of the Arm" domain $(\mathrm{p}=0.01)$. SPADI pain (area $=0.808, \mathrm{p}<0.001)$ and disability (area $=0.885, \mathrm{p}<0.001)$ were the questionnaires that better separated the groups, with cutoff points for SPADI pain $\leq 38$ (sensitivity $66.7 \%$, specificity $79.17 \%$ ) and SPADI disability $\leq 41.25$ (sensitivity 88.3\%, specificity 91.7\%). Conclusions: In patients undergoing conservative treatment and radiotherapy, it was possible to use SPADI and BCTOS to diagnose functional sequelae, since they were able to differentiate the previously known groups in all assessment domains, and SPADI was able to better identify the groups. This fact facilitates the use of these questionnaires in clinical practice, aiming at assessing the sequelae associated with treatment. 